\title{
Mortality in Children with Severe Hand, Foot and Mouth Disease in Guangxi, China
}

\author{
ZHI-Yong YANG ${ }^{1}$, XiU-QI Chen ${ }^{1}$, Dan SUN ${ }^{2}$ ANd DAN WeI ${ }^{1}$ \\ From the Departments of Pediatrics, ${ }^{1}$ The First Affiliated Hospitalof Guangxi Medical University, Nanning, Guangxi, and ${ }^{2}$ The Third \\ People's Hospital of Bengbu,Bengbu, Anhui; China.
}

Correspondence to: Dr Dan Wei, First Affiliated Hospital of Guangxi Medical University, Nanning, Guangxi, China. weidanpicu@163.com.

Received: July 27,2016;

Initial review: November 05, 2016;

Accepted: November 28, 2017.
Objective: To analyze the clinical features of children with hand foot and mouth disease (HFMD) who died. Methods: 331 deaths due to HFMD between 2010 and 2014 were included in this retrospective study; 15 autopsies were performed. Results: Most deaths were seen in children aged below $3 \mathrm{y}$, and with enterovirus 71 infection $(91 \%)$. The mean (SD) duration of HFMD from onset to death was 3.7(2.9) d. The mean (SD) age of fast progressors (from onset to death less than 4 days) was 17.4 (9.2) mo. Most of them were diagnosed as stage 3 and stage 4 of HFMD. Various pathological changes were observed in brain after autopsy, especially in brain stem and medulla. Conclusions: The brain stem encephalitis with the neurotropism of enteroviruses seems to be the main contributor to the death in HFMD.

Keywords: Complications, Enterovirus, Epidemiology, Mortality.

Published online: December 14, 2017. PII:S097475591600098

$\mathrm{H}$ and, foot and mouth disease (HFMD) is a highly contagious disease caused by enterovirus (EV) infection [1]. HFMD is usually benign, but severe cases sometimes progress quickly and may cause mortality [2]. Patients may die in a short duration once they progress to pulmonary edema, pulmonary hemorrhage or cardiopulmonary failure [3]. We analyzed the clinical and pathological features from case records of 331 deaths due to severe HFMD in Guangxi province of China.

\section{Methods}

This retrospective study included 331 deaths due to HFMD, which were reported to the Center for Disease Control (CDC) of Guangxi province from 2010 to 2014. The patients' medical history was provided by Guangxi CDC. The diagnostic criteria and clinical stages were based on the guidelines of Ministry of Health for HFMD and the "Clinical Management of EV71". Throat and anal swabs were collected for the detection of enterovirus and coxsackie viruses. The clinical stages were classified as: Stage 1 - vesicular lesions on hand, foot and mouth; Stage 2 - neurological involvement; Stage 3 - early stage of cardiopulmonary failure; Stage 4 - cardiopulmonary failure; and Stage 5-recovery period [4].

The 331 deaths were distributed in all municipal hospitals in Guangxi province. The clinical data were collected through review of hospital records. The data were collected by three people with a standard data extraction form, and disagreements were resolved by discussion. The form included patients' information such as age, sex, date and time of registry to hospitals, date and time of death, duration from onset to each major complication, duration from each complication to death, clinical features, white blood count (WBC) and blood glucose. Autopsy with the consent of the parents was performed in 15 cases. Based on the duration from onset to death, these cases were classified into slow (equal or more than 4 days) or fast progressors (less than 4 days).

\section{Results}

Among 331deaths, 209 were males (M: $\mathrm{F}=1.71: 1)$. The age ranged from 4 months to 6 years, and 291(87.9\%) were aged below 3 years. $3.0 \%, 27.2 \%, 28.4 \%$ and $41.4 \%$ of patients were diagnosed as stage 1 , stage 2 , stage 3 and stage 4, respectively at the time of registry to the hospitals. The mean (SD) duration from onset of disease to death was 3.7 (2.2) days. The mean (SD) duration from stage 1 to stage 2 was 43.6 (27.2) hours, from stage 2 to stage 3 was 24.6 (16.2) hours, from stage 3 to stage 4 was $3.9(1.5)$ hours, and from stage 4 to death was 4 (1.8) hours. The mean (SD) age of fast progressors was 17.4 (9.2) months, and most of them were diagnosed as stage 3 or stage 4 at registry. The neurological and cardiopulmonary symptoms of these cases are summarized in Table $\mathbf{I}$.

The mean (SD) age of fast progressors was 
significantly lower than that of slow progressors [17.4 (9.2) vs 26.2 (12.7) mo; $P<0.01]$.

301 patients $(90.9 \%)$ were infected by EV71, nine $(2.7 \%)$ cases were infected by Coxsackie A16 and two $(0.6 \%)$ cases were infected by both EV 71 and Coxsackie A 16 viruses. Nineteen $(5.7 \%)$ cases tested negative for both EV 71 and Coxsackie A 16 virus.

Autopsy in all 15 cases showed similar pathological characteristics. The major pathological changes found in the central nervous system (CNS) were: congestion on the surface of cerebrum, obscured cerebral sulcus, brain stem edema, neuronal necrosis, and neuronophagia and colloid deposition in the brain stem. All lung specimens had pink fluid in alveolar space. Alveolar wall was thickened and widened with interstitial fibrosis, hemangiectasis, and congestion. No obvious infiltration by inflammatory cells was seen in heart.

\section{DiscuSSION}

In the present study, we found that most of the deaths in HFMD occurred in younger children ( $\leq 3$ years). These patients had tachycardia, cyanosis, pale skin, hypertension and dyspnea in stage 3 , and pulmonary edema, pulmonary hemorrhage, low blood pressure and bradycardia in stage 4 .

Our findings were consistent with previous reports suggesting that EV71 was more likely to cause neurological impairment, which could lead to severe cases [5-7]. Previous studies showed that patients had increased heart rate and hypertension (stage 3) before cardiopulmonary failure (stage 4 ). These patients may have bradycardia and hypotension leading to cardiopulmonary failure [8]. There was no evidence of viral myocarditis in children in present series, and staining for EV71 antigen was negative in the myocardium and lungs. A previous study also showed that neurogenic pulmonary edema associated with brainstem parenchymal damage, which may be not due to direct virus damage or myocarditis-induced viral damage [9]. However, another study [10] suggested that the invasion of spinal cord and medulla by EV71 contributed to pulmonary edema and other respiratory complications. Kao, et al. [11] suggested that pulmonary edema may result from a sympathetic over-activation. The major limitation of this data is its retrospective nature. Moreover, the data were limited to a single province of China. Autopsy was performed in less than $5 \%$ of cases, thus limiting the generalizability of the findings.

In conclusion, most cases of HFMD had brainstem encephalitis. Close observation on clinical manifestations such as neurological symptoms is critical to assess the severity and prognosis of the disease. Severe HFMD should be diagnosed earlier before stage 3 and receive proper treatment to reduce the mortality.

Contributors: ZYY and XQC: collected the data and wrote the manuscript, and should be considered co-first authors; DS: collected the data and edited the manuscript; DW: contributed to conception and design of study, and revised the manuscript.

Funding: None; Competing interest: None stated.

\section{REFERENCES}

1. McMinn PC. An overview of the evolution of enterovirus 71 and its clinical and public health significance. FEMS Microbiol Rev. 2002;26:91-107.

2. Huang CC, Liu CC, Chang YC, Chen CY, Wang ST, Yeh TF. Neurologic complications in children with enterovirus 71 infection. New England J Med. 1999;341:936-42.

TABLE I Symptoms and Signs Before Death in Hand-Foot-Mouth Disease $(N=331)$

\begin{tabular}{llll}
\hline Symptoms/Signs & $N(\%)$ & Symptoms/Signs & $N(\%)$ \\
\hline Nervous system & & Cardiovascular system & $310(93.7)$ \\
Lethargy & $280(84.6)$ & Tachycardia & $310(93.7)$ \\
Vomiting & $228(68.9)$ & Cyanosis & $164(69.2)$ \\
Irritability & $149(44.1)$ & Low blood pressure & $142(58.9)$ \\
Limb trembling & $127(38.3)$ & Hypertension & $107(32.3)$ \\
Dysphoria & $116(35.1)$ & Bradycardia & $265(80.1)$ \\
Seizure & $103(31.1)$ & Respiratorysystem & $263(79.5)$ \\
Coma & $94(28.4)$ & Expectoration & $188(56.8)$ \\
Eyes stare & $65(19.6)$ & Dyspnea & Crackles \\
Neck rigidity & $54(16.3)$ & & \\
Pale Skin & $210(63.4)$ & & \\
\hline
\end{tabular}

The HFMD children had multiple symptoms and hence the percentages add up to $>100 \%$. 


\section{What This Study Adds?}

- Most deaths due to HFMD occur in children <3 yr of age, and usually due to neurological and cardiac complications.

3. Ho M, Chen ER, Hsu KH, Twu SJ, Chen KT, Tsai SF, et al. An epidemic of enterovirus 71 infection in Taiwan. Taiwan Enterovirus Epidemic Working Group. New England J Med. 1999;341:929-35.

4. Health GOotMo. HFMD diagnosis and treatment guidelines. Available from: URL: http:// www.nhfpc.gov.cn/mohyzs/s3586/201004/46884.shtml. Accessed April 21, 2010.

5. Schmidt NJ, Lennette EH, Ho HH. An apparently new enterovirus isolated from patients with disease of the central nervous system. J Infect Dis. 1974;129:304-9.

6. Chang LY, Huang LM, Gau SS, Wu YY, Hsia SH, Fan TY, et al. Neurodevelopment and cognition in children after enterovirus 71 infection. New England $J$ Med. 2007;356:1226-34.

7. Wong KT, Munisamy B, Ong KC, Kojima H, Noriyo N, Chua KB, et al. The distribution of inflammation and virus in human enterovirus 71 encephalomyelitis suggests possible viral spread by neural pathways. J Neuropathol Experimental Neurol. 2008;67:162-9.

8. Long L, Gao LD, Hu SX, Luo KW, Chen ZH, Ronsmans $\mathrm{C}$, et al. Risk factors for death in children with severe hand, foot, and mouth disease in Hunan, China. Infect Dis. 2016;48:744-8.

9. Wang Z, Nicholls JM, Liu F, Wang J, Feng Z, Liu D, et al. Pulmonary and central nervous system pathology in fatal cases of hand foot and mouth disease caused by enterovirus A71 infection. Pathology. 2016;48:267-74.

10. Chang LY, Huang YC, Lin TY. Fulminant neurogenic pulmonary oedema with hand, foot, and mouth disease. Lancet. 1998;352:367-8.

11. Kao SJ, Yang FL, Hsu YH, Chen HI. Mechanism of fulminant pulmonary edema caused by enterovirus 71 . Clin Infect Dis. 2004;38:1784-8. 